\title{
A Comparison of the Antifungal Activity of Herbal Toothpastes against Other Brands of Toothpastes on Clinical Isolates of Candida albicans and Candida dubliniensis
}

\author{
Arjuna N.B. Ellepola ${ }^{a}$ Zia U. Khan ${ }^{b}$ Rachel Chandy ${ }^{b}$ Leeba Philip ${ }^{a}$ \\ ${ }^{a}$ Faculty of Dentistry and ${ }^{b}$ Department of Microbiology, Faculty of Medicine, Health Sciences Center, \\ Kuwait University, Jabriya, Kuwait
}

Key Words

Candida $\cdot$ Toothpaste $\cdot$ Antifungal effect

\begin{abstract}
Objectives: To evaluate the anti-Candida activity on Candida albicans and Candida dubliniensis species of 2 herbal and 7 other brands of toothpastes commonly used in Kuwait. Materials and Methods: Antifungal activity was determined by agar diffusion test on 65 isolates of $C$. albicans and 21 isolates of $C$. dubliniensis for each toothpaste. A uniform quantity of toothpaste was filled into wells punched into Sabouraud dextrose agar medium plates inoculated with the test isolates, incubated at $37^{\circ} \mathrm{C}$; inhibition zone diameters were read after 24 h. Results: The mean inhibition zone diameters ranged between 12 and $23 \mathrm{~mm}$ for $C$. albicans and between 12 and $27 \mathrm{~mm}$ for $C$. dubliniensis. A herbal toothpaste brand manufactured in the Middle Eastern region (United Arab Emirates) consisting of many herbal ingredients compared to other brands was found to be the most active $(p<0.001)$ against both Candida species tested, which also demonstrated higher inhibitory activity against $C$. dubliniensis isolates compared to C. albicans. Conclusions: The herbal toothpaste brand presented significant anticandidal activity over conventional toothpastes and may be useful in reducing the pathogenic potential of Candida species.
\end{abstract}

Copyright $\odot 2011$ S. Karger AG, Base

\section{KARGER}

Fax +41613061234 E-Mail karger@karger.ch www.karger.com

\section{Introduction}

The oral cavity is known to harbor a large number of microorganisms which coexist with one another as normal microbiota. Among Candida species, Candida albicans is the predominant species normally occurring in the oral cavity [1]. However, under compromised conditions, colonizing $C$. albicans may give rise to different pathologic conditions ranging from acute pseudomembranous thrush to more chronic forms which may persist for a longer period despite treatment [2]. A number of factors such as immunosuppression, antibiotic/radiation therapy, nutritional deficiencies, dental prostheses, high carbohydrate diet, diabetes mellitus, heavy cigarette smoking or poor oral hygiene, which tend to enhance Candida colonization or infection, have been identified [2]. Candida dubliniensis is closely related to C. albicans in evolutionary terms, sharing its properties of commensalism and opportunistic infection. A recent study has shown that there is genomic similarity between these two species as well [3]. In addition to C. albicans, there is current evidence to suggest that $C$. dubliniensis isolates are also involved in the pathogenesis of oral candidal infection in many clinical scenarios $[4,5]$. Further evidence suggests that $C$. dubliniensis can disseminate to other sites as it has been isolated from urine and blood specimens $[6,7]$. 
In recent years, many brands of toothpastes containing different formulations have become available claiming to improve oral hygiene, reduce plaque, gingival inflammation or dental caries [8]. Despite the wide spectrum of antibacterial activity of different toothpaste formulations [8], information on their antifungal properties is scarce $[9,10]$, and none of these studies have determined the anti-candidal activity of toothpastes on $C$. dubliniensis isolates. Hence, we investigated the antiCandida activity on oral as well as nonoral C. albicans and C. dubliniensis isolates of 2 toothpastes consisting of herbal extracts and compared these 2 with 7 other brands of toothpastes commonly used in Kuwait.

\section{Materials and Methods}

Ethical clearance was granted by the Health Sciences Center Ethical Clearance Committee, Faculty of Medicine, Kuwait University. Like other Candida species, C. dubliniensis is part of microbiota with a preference to colonize the oral cavity, has a potential to spread to other anatomic sites and may even cause bloodstream or disseminated infection $[6,7]$. For this reason, we also included C. dubliniensis and C. albicans isolates from sources other than oral cavities, namely blood and urine, in our study.

\section{Isolation and Identification}

A total of 86 isolates of Candida species recovered from various clinical specimens and identified by phenotypic and molecular characteristics were included in the study. The specimens used in the study included 49 oral isolates of C. albicans out of 65 isolates and 20 oral isolates of C. dubliniensis out of 21 isolates. The rest of the non-oral isolates were from blood and urine. All the yeast isolates were tested for germ tube formation. Thereafter, the colony characteristics were observed using CHROMagar Candida medium (Becton Dickinson and Company, Sparks, Md., USA) and were provisionally classified as $C$. albicans. All the isolates were further identified by the Vitek 2 yeast ID system (bioMérieux). The identity of $C$. dubliniensis was confirmed by production of rough colonies with hyphal fringes and chlamydospores on simplified sunflower seed agar [11]. The species-specific identity was investigated by seminested PCR amplification of the internal transcribed spacer-2 region of rDNA and further confirmed by direct DNA sequencing of the internal transcribed spacer region of the rDNA as described previously [12].

\section{Susceptibility Testing}

Nine different brands of toothpaste (table 1) were purchased from the local market. The particulars of ingredients included in each toothpaste formulation by the manufacturers are provided in table 1. Each brand has been identified by serial number, brand name and country of production. The antifungal activity of the toothpastes was determined by agar diffusion test. Growth from freshly subcultured isolates was suspended in $5 \mathrm{ml}$ of sterile saline to obtain a turbidity of $0.5 \mathrm{McF}$ arland standard. Using a sterile swab, the Sabouraud dextrose agar plates (Difco TM, Beckton Dickinson and Company), each containing $60 \mathrm{ml}$ of medium,

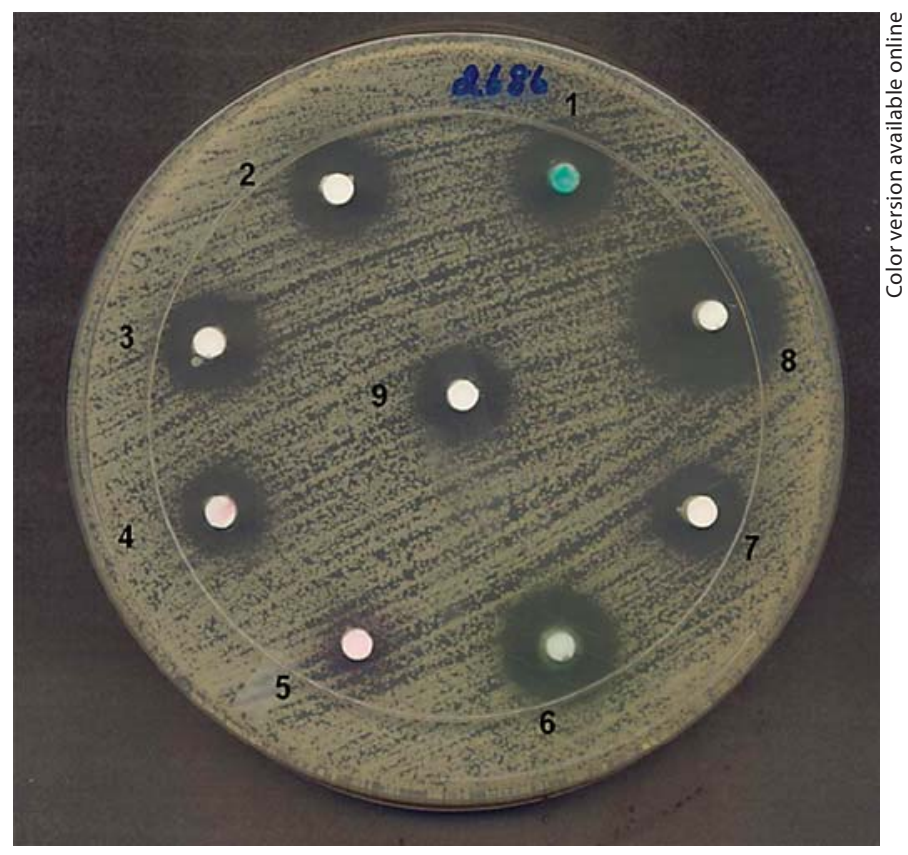

Fig. 1. Inhibition zones of different toothpastes (numbered 1-9) against a C. dubliniensis isolate on a Sabouraud agar plate. Note the maximum zone of inhibition with toothpaste No. 8 .

were evenly inoculated with the yeast suspension. A uniform quantity of each toothpaste preparation was filled into $6-\mathrm{mm}$ diameter wells punched into the pre-inoculated plates. The plates were incubated at $37^{\circ} \mathrm{C}$ and readings for inhibition zone diameters were taken after $24 \mathrm{~h}$. Measurement of the inhibition zone was read independently by 1 coauthor (R.C.) and 2 other laboratory microbiologists who were unaware of the type of coded toothpaste filled into the 6-mm diameter wells. Each Candida isolate was tested separately for each of the 9 toothpaste brands.

\section{Statistical Analysis}

Statistical analysis was done by one-way ANOVA and the Bonferroni multiple comparison test to compare the inhibitory effect of different brands of toothpaste on Candida species and to compare the inhibitory effect among different brands of toothpaste. A $p$ value of $<0.05$ was considered significant. However, for illustration, the results are presented as means and standard deviations.

\section{Results}

The inhibition zones observed for one such C. dubliniensis isolate against all 9 toothpaste brands used in the study are shown in figure 1 . Different toothpaste brands showed varying inhibitory activity against the two Candida species tested (table 2). The mean inhibition zone diameters of the 9 toothpaste brands ranged between 12 
Table 1. Ingredient details of formulations of different brands of toothpaste tested for anti-Candida activity

\begin{tabular}{lll}
\hline $\begin{array}{l}\text { Serial } \\
\text { No. }\end{array}$ & $\begin{array}{l}\text { Brand name and country } \\
\text { of manufacture }\end{array}$ & Ingredients used in different brands of toothpaste \\
\hline 1 & Signal, Saudi Arabia & $\begin{array}{l}\text { sorbitol, water, hydrated silica, PEG-32, sodium lauryl sulfate, flavor, cel- } \\
\text { lulose gum, sodium fluoride, sodium saccharin, PEG-40, hydrogenated } \\
\text { castor oil, phenoxyethanol, glycerin, limonene, CI 19140, CI 42090, } \\
\text { CI 74260 }\end{array}$
\end{tabular}

\begin{tabular}{lll}
\hline 2 Elgydium, France & $\begin{array}{l}\text { chlorohexidine digluconate } 0.004 \text { g, calcium carbonate } 25 \text { g, sodium lauryl } \\
\text { sulfate, glycerol, colloidal silica, amorphous hydrated precipitated silica, } \\
\text { carrageenate, sodium carboxymethyl cellulose, titanium oxide, methyl } \\
\text { parahydroxybenzoate, propyl parahydroxybenzoate, saccharin sodium, } \\
\text { artificial flavoring, water }\end{array}$ \\
\hline $3 \quad$ Colgate, Saudi Arabia & $\begin{array}{l}\text { sodium monofluorophosphate } 0.76 \%, \text { sodium fluoride } 0.1 \%, \text { dicalcium } \\
\text { phosphate dihydrate, aqua, sorbitol, glycerin, PEG-12, sodium lauryl sul- } \\
\text { fate, aroma, cellulose gum, sodium monofluorophosphate, tetrasodium } \\
\text { pyrophosphate, sodium saccharin, sodium fluoride, limonene; contains } \\
\text { no sugar }\end{array}$ \\
\hline
\end{tabular}

4 Aquafresh, UK sodium fluoride $0.306 \% \mathrm{w} / \mathrm{w}$, aqua, hydrated silica, sorbitol, glycerine, PEG-6, sodium lauryl sulfate, aroma, xanthan gum, titanium dioxide, carrageenan, sodium fluoride, sodium saccharin, limonene, CI 73360, CI 74260, CI 74160

\begin{tabular}{lll}
\hline 5 & Sensodyne, UK & strontium chloride hexahydrate 10\% \\
\hline 6 & Close up, Saudi Arabia & $\begin{array}{l}\text { sodium fluoride } 0.32 \% \text {, triclosan, sorbitol, silica, water, PEG-32, sodium } \\
\text { lauryl sulfate, mix of flavors, cellulose gum, sodium saccharin, tocopheryl } \\
\text { acetate, CI 47005, CI 42090 }\end{array}$ \\
\hline $7 \quad \begin{array}{l}\text { Miswak Herbal, } \\
\text { United Arab Emirates }\end{array}$ & $\begin{array}{l}\text { calcium carbonate, sorbitol, treated water, silica, sodium lauryl sulfate, } \\
\text { flavor, miswak extract, sodium carboxymethyl cellulose and/or sodium } \\
\text { carrageenate, sodium silicate, sodium benzoate, glycerine, sodium sac- } \\
\text { charin }\end{array}$ \\
\hline Dabur Herbal, & $\begin{array}{l}\text { sodium fluoride 0.32\%, sorbitol, triclosan 0.20\%, treated water, silica, } \\
\text { polyethylene glycol 1500, sodium lauryl sulfate, miswak extract, neem ex- } \\
\text { tract, lemon extract and/or lemon oil, flavor containing mint, clove, basil } \\
\text { and other natural oils, glycerine, titanium dioxide, sodium carrageenate } \\
\text { and/or sodium carboxymethyl cellulose, trisodium orthophosphate, so- } \\
\text { dium saccharin, citric acid, FD\&C Yellow No. 5, FD\&C Blue No. 1 }\end{array}$ \\
\hline Salz F, Japan & $\begin{array}{l}\text { sodium chloride, dipotassium glycyrrhizinate, sorbitol, propylene glycol, } \\
\text { sodium lauryl sulfate, calcium carbonate, ethyl paraben, butyl paraben, } \\
\text { sugar-free }\end{array}$ \\
\hline &
\end{tabular}

and $23 \mathrm{~mm}$ for C. albicans ( $<<0.001)$ and between 12 and $27 \mathrm{~mm}$ for C. dubliniensis ( $\mathrm{p}<0.001$ ). Based on inhibition zone diameter, the toothpaste which contained as its ingredients many herbal extracts from the miswak tree (Salvadora persica/arak tree), neem tree (Azadirachta indica), cloves (Syzygium aromaticum), basil (Ocimum basilicum) and some other natural oils (No. 8) was the most active against the two Candida species. This brand showed a higher inhibitory activity against $C$. dubli- niensis $(27 \pm 3.7 \mathrm{~mm})$ than against C. albicans $(23 \pm$ $3.9 \mathrm{~mm}$ ). A nonherbal toothpaste (No. 9) also showed a higher inhibitory activity against $C$. albicans $(20 \pm$ $4.1 \mathrm{~mm})$. However, the inhibitory effect of this brand against $C$. dubliniensis was much lower when compared to the herbal brand (18 \pm 3.6 vs. $27 \pm 3.7 \mathrm{~mm})$. The inhibitory effect of a nonherbal toothpaste containing only strontium chloride hexahydrate (No. 5) had the lowest inhibitory effect on both Candida species (table 2). The 
Table 2. Antifungal activity of different brands of toothpaste tested against clinical isolates of Candida species

\begin{tabular}{lll}
\hline \multirow{2}{*}{$\begin{array}{l}\text { Serial } \\
\text { No. }\end{array}$} & \multicolumn{2}{c}{ Mean zone of inhibition of Candida, $\mathrm{mm}$} \\
\cline { 2 - 3 } & C. albicans $(\mathrm{n}=65)$ & C. dubliniensis $(\mathrm{n}=21)$ \\
\hline 1 & $16 \pm 1.8$ & $17 \pm 2.1$ \\
2 & $17 \pm 2.4$ & $18 \pm 2.9$ \\
3 & $18 \pm 2.5$ & $19 \pm 3.5$ \\
4 & $14 \pm 1.9$ & $15 \pm 3.0$ \\
5 & $12 \pm 3.2$ & $12 \pm 2.8$ \\
6 & $17 \pm 2.5$ & $20 \pm 2.9$ \\
7 & $17 \pm 2.8$ & $19 \pm 3.2$ \\
8 & $23 \pm 3.9$ & $27 \pm 3.7$ \\
9 & $20 \pm 4.1$ & $18 \pm 3.6$ \\
\hline
\end{tabular}

Values denote means \pm SD.

herbal toothpaste (No. 8) was the only brand which produced a significant inhibitory effect on both Candida species even when compared with all other brands of toothpastes used in the study $(\mathrm{p}<0.001)$. The inhibitory activity of the nonherbal toothpaste No. 9 against C. albicans was also significantly higher when compared to the inhibitory effect of other toothpaste brands $(\mathrm{p}<$ 0.001 ). However, the inhibitory effect of this brand on $C$. dubliniensis was not significantly different when compared to the inhibitory effect of other toothpaste brands. The inhibitory effect of a toothpaste containing only strontium chloride hexahydrate (No. 5), which had the lowest inhibitory effect on both Candida species, was significantly lower $(\mathrm{p}<0.001)$ when compared to the higher inhibitory activity of other toothpaste brands.

\section{Discussion}

Candida species occupy a predominant place in the etiology of oral fungal infections [2], and recent studies have confirmed the etiologic role of Candida species in endodontic infections [13]. Although the dorsum of the tongue is believed to be the primary oral habitat of C. albicans, other sites may also be colonized [2], including the buccal mucosa, palate, angle of the mouth and tooth structures such as the dentin and the root $[2,13,14]$. Candida species can also colonize subgingival pockets and may cause gingivitis particularly in those using dental prostheses $[15,16]$. Besides C. albicans, other yeast species including C. dubliniensis have also been isolated from the oral cavity in both healthy and compromised individuals $[4,5]$.

Candida and Antifungal Activity of

Toothpaste
In recent years, a number of toothpaste preparations containing herbal ingredients that may be beneficial by improving oral health have been developed. One such ingredient is an extract of aqueous miswak, which possesses antibacterial activity against Streptococcus mutans [17], Actinobacillus actinomycetemcomitans [18], Bacteroides species [19] and some other oral microorganisms associated with periodontitis and caries [20], although it did not show any inhibitory effect on Staphylococcus aureus and Staphylococcus epidermidis [17]. A few studies carried out to determine the anti-Candida activity of miswak extracts have yielded inconsistent results [17]. For instance, in one study it was found that miswak extract exhibited fungistatic activity against $C$. albicans in concentrations of $15 \%$ and above up to $48 \mathrm{~h}$ as determined by turbidity measurements [21]. In a another study, however, no inhibitory activity against $C$. albicans was observed even at a concentration as high as 50\% [17]. Hence, the information on the active ingredients possessing antibacterial or antifungal activity is inconsistent.

The other herbal ingredient known to demonstrate inhibitory activity against Candida species is neem. Neem and its products (seeds and oil) have been shown to possess antifungal properties against phytopathogenic filamentous (mould) fungi such as Fusarium species, Drechslera species, Alternaria species and dermatophytes $[22,23]$. Some of the latter species are also opportunistic human pathogens. The chemical nature of some of the ingredients that exhibit antifungal activity in neem extracts against filamentous fungi have been characterized [22], but information on their anti-Candida activity is lacking. Other herbal preparations such as oils obtained from clove and basil have also shown antifungal activity against a number of fungi including C. albicans [24]. A contact time of about $1 \mathrm{~min}$ with clove oil is sufficient to eliminate an inoculum of $1 \times 10^{6}$ colony-forming units per milliliter of C. albicans. In addition, extracts of chamomile, Echinacea, peppermint and rhatany have also been reported to possess some antifungal properties [25].

In the current study, the herbal toothpaste brand (No. 8) commonly used in Kuwait showed a significantly higher activity against $C$. dubliniensis than against $C$. albicans, most probably due to a synergistic effect of the herbal constituents (at least 6) included in this formulation. The other toothpastes containing non-herbal ingredients (table 1) also elicited anti-Candida activity, most probably due to the antifungal properties documented in some of these ingredients. For instance, it has recently been suggested that triclosan blocks lipid biosynthesis by specifically inhibiting the enzyme enoyl-acyl carrier pro-

Med Princ Pract 2011:20:112-117 115 
tein reductase $[8,10]$. Chlorhexidine suppresses many pathogenic attributes of Candida species [26]. Fluorides have also demonstrated some antibacterial and antifungal effects such as metabolic interference and reduction of dental plaque acidogenicity [10]. Similar antifungal activity of toothpastes containing triclosan and sodium monofluorophosphate/sodium fluoride on Candida species has previously been reported [8-10]. The non-herbal toothpaste containing only strontium chloride hexahydrate (No. 5) had the lowest inhibitory effect on both Candida species, which may be due to the presence of a single ingredient in its formulation.

The inhibitory zone diameters observed in our study for the herbal toothpaste with the highest inhibitory effect range of 23-27 $\mathrm{mm}$ were within the range of a toothpaste containing monofluorophosphate (19-36 $\mathrm{mm}$ ) [9], but not of another herbal toothpaste (35$50 \mathrm{~mm}$ ) [10]. These differences in inhibitory effect may be attributed to the ingredients used in the toothpaste formulations as well as to the susceptibility of Candida species used in the aforementioned studies. However, none of the previous studies $[9,10]$ tested the anti-candidal effect of toothpastes on C. dubliniensis isolates. $C$. dubliniensis is now well recognized as an opportunistic pathogen associated with oral candidiasis. Invasive $C$. dubliniensis infections have also been described in immunocompromised HIV-seropositive individuals and in patients with gynecological malignancies, chronic myelogenous leukemia, liver disease or myelodysplastic syndromes [27, 28]. Resistance to fluconazole has also been observed in C. dubliniensis isolates obtained from AIDS patients, and stable fluconazole resistance can be readily induced in C. dubliniensis following exposure to the drug in vitro [29]. In addition, it was recently reported that breakthrough C. dubliniensis fungemia occurred in a patient during prolonged exposure to voriconazole [30]. In this context the antifungal effect of the herbal brand of toothpaste, especially on C. dubliniensis isolates as shown in our study, would be of interest as it may provide possible beneficial effects by reducing the pathogenic potential of this species in the oral cavity in susceptible individuals.

The currently available toothpaste formulations that are recommended by oral healthcare providers for their ability to control plaque, thereby preventing dental caries and periodontal disease, are mostly those active against bacterial pathogens. A number of antibacterial agents, such as bromochlorophene, phenolic compounds, tin and zinc salts, chlorhexidine and, more recently, triclosan have been used as therapeutic agents in toothpastes and mouthwashes to reduce plaque formation, gingivitis and calculus [8]. In this context the current study is noteworthy as it contributes to the understanding of the effectiveness of different toothpaste preparations against commonly encountered oral Candida species. Although none of the toothpaste brands investigated was developed for use as an antifungal formulation, the information provided herein, especially the anti-Candida property of the toothpaste containing herbal extracts, could be valuable to oral healthcare providers in recommending a toothpaste to reduce the burden of Candida in the oral cavity in individuals susceptible to oral candidosis. Furthermore, this herbal toothpaste may be used as an alternative to conventional formulations by individuals who have an interest in naturally based products. Further clinical studies using a larger number of oral Candida isolates are warranted to confirm these findings in vitro and to allocate herbal toothpastes to be used as therapeutic agents in oral candidosis. In addition, as isolates from non-oral sources were also used in this study, it suggests that the anticandidal properties of ingredients in toothpastes may have a wider applicability and could be considered for the future development of anti-candidal drugs principally for topical application.

\section{Conclusion}

The herbal toothpaste presented significant anti-candidal activity against two important Candida species associated with oral candidosis compared to other conventional formulations. Hence, the herbal brand may be considered to be used as an alternative to conventional formulations in patients susceptible to oral candidosis.

\section{Acknowledgments}

The technical help received from Daad Farhat and Salwa Al-Hajri is appreciated and thankfully acknowledged. The work in this study was supported by Kuwait University Research Grant No. DB01/07. 


\section{References}

$\checkmark 1$ Cannon RD, Chaffin WL: Oral colonization by Candida albicans. Crit Rev Oral Biol Med 1999; 10:359-383.

$\checkmark 2$ Samaranayake LP, Cheung LK, Samaranayake $\mathrm{YH}$ : Candidiasis and other fungal diseases of the mouth. Dermatol Ther 2002;15: 251-269.

-3 Moran G, Stokes C, Thewes S, Hube B, Coleman DC, Sullivan D: Comparative genomics using Candida albicans DNA microarrays reveals absence and divergence of virulenceassociated genes in Candida dubliniensis. Microbiol 2004;150:3363-3382.

4 Manfredi M, McCullough MJ, Al-Karaawi ZM, Hurel SJ, Porter SR: The isolation, identification and molecular analysis of Candida spp isolated from the oral cavities of patients with diabetes mellitus. Oral Microbiol Immunol 2002;17:181-185.

$\checkmark 5$ Peltroche-Llacsahuanga $\mathrm{H}$, Döhmen $\mathrm{H}$, Haase G: Recovery of Candida dubliniensis from sputum of cystic fibrosis patients. Mycoses 2002;45:15-18.

-6 Brandt ME, Harrison LH, Pass M, Sofair AN, Huie S, Li RK, Morrison CJ, Warnock DW, Hajjeh RA: Candida dubliniensis fungemia: the first four cases in North America. Emerg Infect Dis 2000;6:46-49.

$\checkmark 7$ Cimolai N, Davis J, Trombley C: Candida dubliniensis fungemia and vascular access infection. J Pediatr Hematol Oncol 2002;24: 237-239.

$\checkmark 8$ Moran J, Newcombe RG, Wright P, Haywood J, Marlow I, Addy M: A study into the plaqueinhibitory activity of experimental toothpaste formulations containing antimicrobial agents. J Clin Periodontol 2005;32:841-845.

$\checkmark 9$ Abirami CP, Venugopal PV: In vitro evaluation of antifungal activity of toothpastes. Mycol Med 2005;15:247-249.

$\checkmark 10$ Yigit N, Aktas E, Ayyildiz A: Antifungal activity of toothpastes against oral Candida isolates. J Mycol Med 2008;18:141-146.
11 Khan ZU, Ahmad S, Mokaddas E, Chandy R: Simplified sunflower (Helianthus annuus) seed agar for differentiation of Candida dub liniensis from Candida albicans. Clin Microbiol Infect 2004;10:590-592.

12 Ahmad S, Mokaddas E, Al-Sweih N, Khan ZU: Phenotypic and molecular characterization of Candida dubliniensis isolates from clinical specimens in Kuwait. Med Princ Pract 2005; 14:177-183.

13 Siqueira JF, Sen BH: Fungi in endodontic infections. Oral Surg Oral Med Oral Pathol Oral Radiol Endod 2004;97:632-641.

14 Jacobs LS, Flaitz CM, Nicholos CM, Hicks MJ: Role of dentinal carious lesions in the pathogenesis of oral candidiasis in HIV infection. J Am Dent Assoc 1998;129:187-194.

15 Jewtuchowicz VM, Brusca MI, Mujica MT Gliosca LA, Finquelievich JL, Lovannitti CA, Rosa AC: Subgingival distribution of yeast and their antifungal susceptibility in immunocompetent subjects with and without dental devices. Acta Odontol Latinoam 2007;20:17-22.

16 Aas JA, Barbuto SM, Alpagot T, Olsen I, Dewhirst FE, Paster BJ: Subgingival plaque microbiota in HIV positive patients. J Clin Periodontol 2007;34:189-195

17 Almas K, Al-Bagieh NH: The antimicrobial effects of bark and pulp extracts of miswak Salvadora persica. Biomed Lett 1999;60:7175 .

18 Al-Otaibi M, Al-Harthy M, Gustafsson A, Johansson A, Claesson R, Angmar-Månsson B: Subgingival plaque microbiota in Saudi Arabians after use of miswak chewing stick and toothbrush. J Clin Periodontol 2004;31: 1048-1053.

19 Rotimi VO, Mosadomi HA: Effect of crude extracts of nine African chewing sticks on oral anaerobes. J Med Microbiol 1987;23:5560.

20 Sofrata AH, Claesson RLK, Lingström PK, Gustafsson AK: Strong antibacterial effect of miswak against oral microorganisms associated with periodontitis and caries. J Peridontol 2008;79:1474-1479.
21 Al-Bagieh NH, Idowu A, Salako NO: Effect of aqueous extract of miswak on the in vitro growth of Candida albicans. Microbios 1994; 80:107-113.

>22 Govindachari TR, Suresh G, Gopalakrishnan G, Banumathy B, Masilamani S: Identification of antifungal compounds from the seed oil of Azadirachta indica. Phytoparasitica 1998;26:109-116.

23 Subapriya R, Nagini S: Medicinal properties of neem leaves: a review. Curr Med Chem Anticancer Agents 2005;5:149-156

$\checkmark 24$ Edris AE, Farrag ES: Antifungal activity of peppermint and sweet basil essential oils and their major aroma constituents on some plant pathogenic fungi from the vapor phase. Nahrung 2003;47:117-121.

25 Hili P, Evans CS, Veness RG: Antimicrobial action of essential oils: the effect of dimethylsulphoxide on the activity of cinnamon oil. Lett Appl Microbiol 1997;24:269-275.

26 Ellepola AN, Samaranayake LP: Adjunctive use of chlorhexidine in oral candidosis: a review. Oral Dis 2001;7:11-17.

27 Chan-Tack KM: Fatal Candida dubliniensis septicemia in a patient with AIDS. Clin Infect Dis 2005;40:1209-1210.

$>28$ Meis JF, Ruhnke M, de Pauw BE, Odds FC, Siegert W, Verweij PE: Candida dubliniensis candidemia in patients with chemotherapyinduced neutropenia and bone marrow transplantation. Emerg Infect Dis 1999;5: 150-153.

29 Moran GP, Sanglard D, Donnelly SM, Shanley DB, Sullivan DJ, Coleman DC: Identification and expression of multidrug transporters responsible for fluconazole resistance in Candida dubliniensis. Antimicrob Agents Chemother 1998;42:1819-1830.

30 Fanci R: Breakthrough Candida dubliniensis fungemia in an acute myeloid leukemia patient during voriconazole therapy successfully treated with caspofungin. J Chemother 2009;21:105-107. 